\title{
Estimation of the Accuracy of the Averaging Method for Systems with Multifrequency Perturbations
}

\author{
E. I. Kugushev， T. V.Popova
}

We consider systems of ordinary differential equations whose right-hand sides contain timeperiodic functions with some frequencies. An averaged system is constructed by introduction of additional variables and by step-by-step averaging over these variables. An upper estimate of the deviation of the solution to the initial system from the solution to the averaged system is given. Examples are given of mechanical systems in which vibrations with several frequencies occur and for the analysis of which the statements obtained are applied.

Keywords: averaging method, multifrequency perturbations, vibration frequency

The motion of mechanical systems in which elements performing forced oscillations are present can be described by ordinary differential equations involving time-periodic functions with some frequencies. Examples of such systems include systems on a vibrating base. Reference [1] considers single-frequency systems in which the vibration of the base is described by a timeperiodic function with a large frequency. In this case the equations of motion can be reduced to a form that is standard for the Krylov-Bogolyubov averaging methods [2], and these methods can be used to analyze the motion of the system. The key point of these methods is an estimate of the deviation of the solution to the averaged system from the solution to the initial system. In the multifrequency case the application of averaging methods is complicated by the presence of resonances, i.e., situations where the frequencies are related by rational linear homogeneous relations. The motion of the system in the resonance case can greatly differ from that of an averaged system. However, even in this situation one can apply averaging methods, for example, in the case of separation of oscillations into fast and slow ones or in the case of strong

Received December 24, 2019

Accepted March 15, 2020

This work was supported by the Russian Foundation for Basic Research (project 18-01-00887).

\author{
Eugene I. Kugushev \\ kugushev@keldysh.ru \\ Tatiana V. Popova \\ t.shahova@yandex.ru \\ Lomonosov Moscow State University \\ GSP-1, Leninskie Gory, Moscow, 119991 Russia
}

RUSSIAN JOURNAL OF NONLINEAR DYNAMICS, 2020, 16(2), 379-394 
incommensurability of frequencies. An analysis of the application of averaging methods in multifrequency systems can be found in [3-8].

In this paper, we consider multifrequency systems in "slow time" on a finite time interval. By introducing additional variables, we sequentially construct systems averaged over these variables and give estimates of deviations of solutions to the averaged systems from the solution to the initial system. In proving the statements we use direct integral estimates. But it should be noted that the application of the standard method of change of variables [9] could somewhat shorten the exposition.

\section{Systems with separable right-hand sides}

Consider a system of ordinary differential equations

$$
\dot{\vec{x}}=\vec{g}(\vec{x})+\vec{f}_{1}\left(\vec{x}, \nu_{1} t\right)+\vec{f}_{2}\left(\vec{x}, \nu_{2} t\right), \quad \vec{x} \in \mathbb{R}^{n}, \quad \nu_{1}>0, \nu_{2}>0 .
$$

Let the functions $\vec{g}(\vec{x}), \vec{f}_{1}(\vec{x}, \tau), \vec{f}_{2}(\vec{x}, \tau)$ be continuously differentiable with respect to the variables $\vec{x} \in \mathbb{R}^{n}, \tau \in \mathbb{R}$, the functions $\vec{f}_{1}(\vec{x}, \tau), \overrightarrow{f_{2}}(\vec{x}, \tau)$ be periodic in $\tau$ with period 1 , independent of $\nu_{1}, \nu_{2}$, and let their average on a period in $\tau$ be zero. Averaging the right-hand side of the system (1.1)

$$
\int_{0}^{1} \int_{0}^{1}\left(\vec{g}(\vec{x})+\vec{f}_{1}\left(\vec{x}, \tau_{1}\right)+\vec{f}_{2}\left(\vec{x}, \tau_{2}\right)\right) d \tau_{1} d \tau_{2}=\vec{g}(\vec{x})
$$

we write the averaged system

$$
\dot{\vec{z}}=\vec{g}(\vec{z}), \quad \vec{z} \in \mathbb{R}^{n} .
$$

Statement 1. Suppose that for some $a>0$ and any $\nu_{1}, \nu_{2}>0$ the solutions $\vec{x}\left(t, \nu_{1}, \nu_{2}, \vec{x}_{0}\right)$ and $\vec{z}\left(t, \vec{x}_{0}\right)$ to systems (1.1) and (1.2) with initial conditions $\vec{x}\left(0, \nu_{1}, \nu_{2}, \vec{x}_{0}\right)=\vec{x}_{0}$ and $\vec{z}\left(0, \vec{x}_{0}\right)=$ $=\vec{x}_{0}$, where $\vec{x}_{0}$ lies in some bounded region $U \subset \mathbb{R}^{n}$, exist on the interval $0 \leqslant t \leqslant a$ and lie in some bounded region $V \subset \mathbb{R}^{n}$. Then one can find constants $c_{1}, c_{2}>0$ such that for all $\vec{x}_{0} \in U$ one has

$$
\left|\vec{x}\left(t, \nu_{1}, \nu_{2}, \vec{x}_{0}\right)-\vec{z}\left(t, \vec{x}_{0}\right)\right| \leqslant \frac{c_{1}}{\nu_{1}}+\frac{c_{2}}{\nu_{2}} \quad \text { with } \quad 0 \leqslant t \leqslant a .
$$

Proof. For brevity we will sometimes write the solutions $\vec{x}\left(t, \nu_{1}, \nu_{2}, \vec{x}_{0}\right), \vec{z}\left(t, \vec{x}_{0}\right)$ as $\vec{x}(t), \vec{z}(t)$. We write the initial and the averaged system in the integral form

$$
\vec{x}(t)=\vec{x}_{0}+\int_{0}^{t}\left(\vec{g}(\vec{x}(s))+\vec{f}_{1}\left(\vec{x}(s), \nu_{1} s\right)+\vec{f}_{2}\left(\vec{x}(s), \nu_{2} s\right)\right) d s, \quad \vec{z}(t)=\vec{x}_{0}+\int_{0}^{t} \vec{g}(\vec{z}(s)) d s .
$$

For

$$
\vec{u}\left(t, \nu_{1}, \nu_{2}, \vec{x}_{0}\right)=\vec{x}\left(t, \nu_{1}, \nu_{2}, \vec{x}_{0}\right)-\vec{z}\left(t, \vec{x}_{0}\right)
$$

we have

$$
\vec{u}\left(t, \nu_{1}, \nu_{2}, \vec{x}_{0}\right)=\int_{0}^{t}(\vec{g}(\vec{x}(s))-\vec{g}(\vec{z}(s))) d s+\int_{0}^{t} \vec{f}_{1}\left(\vec{x}(s), \nu_{1} s\right) d s+\int_{0}^{t} \vec{f}_{2}\left(\vec{x}(s), \nu_{2} s\right) d s .
$$

Let us fix an arbitrary $t$ from the interval $[0, a]$ and estimate $\left|\vec{u}\left(t, \nu_{1}, \nu_{2}, \vec{x}_{0}\right)\right|$. Since the functions $\vec{g}(\vec{x}), \vec{f}_{1}(\vec{x}, \tau), \overrightarrow{f_{2}}(\vec{x}, \tau)$ are continuously differentiable with respect to all variables and 
the functions $\vec{f}_{1}(\vec{x}, \tau), \vec{f}_{2}(\vec{x}, \tau)$ are periodic in $\tau$, it follows that in the region $V \subset \mathbb{R}^{n}$ for any $t$, $\nu_{1}, \nu_{2}$ the functions $\vec{g}, \overrightarrow{f_{1}}, \overrightarrow{f_{2}}$ are bounded by some constant $K$ :

$$
|\vec{g}(\vec{x})| \leqslant K, \quad\left|\vec{f}_{1}\left(\vec{x}, \nu_{1} t\right)\right| \leqslant K, \quad\left|\vec{f}_{2}\left(\vec{x}, \nu_{2} t\right)\right| \leqslant K
$$

and satisfy the Lipschitz condition in $\vec{x}$ with some constant $L$ :

$$
\begin{gathered}
\left|\vec{g}(\vec{x})-\vec{g}\left(\vec{x}^{\prime}\right)\right| \leqslant L\left|\vec{x}-\vec{x}^{\prime}\right|, \quad\left|\overrightarrow{f_{1}}\left(\vec{x}, \nu_{1} t\right)-\vec{f}_{1}\left(\vec{x}^{\prime}, \nu_{1} t\right)\right| \leqslant L\left|\vec{x}-\vec{x}^{\prime}\right|, \\
\left|\overrightarrow{f_{2}}\left(\vec{x}, \nu_{2} t\right)-\vec{f}_{2}\left(\vec{x}^{\prime}, \nu_{2} t\right)\right| \leqslant L\left|\vec{x}-\vec{x}^{\prime}\right| .
\end{gathered}
$$

Then, according to (1.5), for the first term on the right-hand side of (1.3) we have

$$
\left|\int_{0}^{t}(\vec{g}(\vec{x}(s))-\vec{g}(\vec{z}(s))) d s\right| \leqslant \int_{0}^{t}|\vec{g}(\vec{x}(s))-\vec{g}(\vec{z}(s))| d s \leqslant L \int_{0}^{t}\left|\vec{u}\left(s, \nu_{1}, \nu_{2}, \vec{x}_{0}\right)\right| d s .
$$

Let us estimate the second term on the right-hand side of (1.3). Let $t_{k}=\frac{k}{\nu_{1}}$, where $k=$ $=0,1, \ldots, m$, and let $m$ be the maximal integer for which $t_{m}=\frac{m}{\nu_{1}} \leqslant t$. It follows from (1.4) that $\left|\vec{g}+\vec{f}_{1}+\vec{f}_{2}\right| \leqslant 3 K$ for any $t, \nu_{1}, \nu_{2}$, and therefore $\left|\dot{\vec{x}}\left(t, \nu_{1}, \nu_{2}, \vec{x}_{0}\right)\right| \leqslant 3 K$. Hence, on the interval $t_{k} \leqslant s \leqslant t_{k+1}$ we have

$$
\left|\vec{x}\left(s, \nu_{1}, \nu_{2}, \vec{x}_{0}\right)-\vec{x}\left(t_{k}, \nu_{1}, \nu_{2}, \vec{x}_{0}\right)\right| \leqslant 3 K\left(s-t_{k}\right)
$$

and, taking (1.5) into account,

$$
\left|\vec{f}_{1}\left(\vec{x}(s), \nu_{1} s\right)-\vec{f}_{1}\left(\vec{x}\left(t_{k}\right), \nu_{1} s\right)\right| \leqslant L\left|\vec{x}(s)-\vec{x}\left(t_{k}\right)\right| \leqslant 3 L K\left(s-t_{k}\right) .
$$

The average of the function $\vec{f}_{1}(\vec{x}, \tau)$ over $\tau$ on a period is zero. Therefore,

$$
\int_{t_{k}}^{t_{k+1}} \vec{f}_{1}\left(\vec{x}\left(t_{k}\right), \nu_{1} s\right) d s=0
$$

Then

$$
\begin{gathered}
\left|\int_{t_{k}}^{t_{k+1}} \vec{f}_{1}\left(\vec{x}(s), \nu_{1} s\right) d s\right|=\left|\int_{t_{k}}^{t_{k+1}}\left(\vec{f}_{1}\left(\vec{x}(s), \nu_{1} s\right)-\vec{f}_{1}\left(x\left(t_{k}\right), \nu_{1} s\right)\right) d s\right| \leqslant \\
\leqslant \int_{t_{k}}^{t_{k+1}}\left|\overrightarrow{f_{1}}\left(\vec{x}(s), \nu_{1} s\right)-\vec{f}_{1}\left(\vec{x}\left(t_{k}\right), \nu_{1} s\right)\right| d s \leqslant 3 L K \int_{t_{k}}^{t_{k+1}}\left(s-t_{k}\right) d s=\frac{3 L K\left(t_{k+1}-t_{k}\right)^{2}}{2}=\frac{3 L K}{2 \nu_{1}^{2}} .
\end{gathered}
$$

In view of the fact that $m \leqslant \nu_{1} t \leqslant \nu_{1} a$, we have

$$
\left|\int_{0}^{t_{m}} \overrightarrow{f_{1}}\left(\vec{x}(s), \nu_{1} s\right) d s\right| \leqslant \sum_{k=0}^{m-1}\left|\int_{t_{k}}^{t_{k+1}} \overrightarrow{f_{1}}\left(\vec{x}(s), \nu_{1} s\right) d s\right| \leqslant \frac{3 m L K}{2 \nu_{1}^{2}} \leqslant \frac{3 a L K}{2 \nu_{1}} .
$$


Since $\left|t-t_{m}\right|<\frac{1}{\nu_{1}}$, we have

$$
\left|\int_{t_{m}}^{t} \vec{f}_{1}\left(\vec{x}(s), \nu_{1} s\right) d s\right| \leqslant \frac{K}{\nu_{1}} .
$$

Thus, for the second term on the right-hand side of (1.3) we obtain

$$
\left|\int_{0}^{t} \overrightarrow{f_{1}}\left(\vec{x}(s), \nu_{1} s\right) d s\right| \leqslant\left|\int_{0}^{t_{m}} \vec{f}_{1}\left(\vec{x}(s), \nu_{1} s\right) d s\right|+\left|\int_{t_{m}}^{t} \vec{f}_{1}\left(\vec{x}(s), \nu_{1} s\right) d s\right| \leqslant \frac{3 a L K}{2 \nu_{1}}+\frac{K}{\nu_{1}}=\frac{d_{1}}{\nu_{1}},
$$

where $d_{1}$ is the constant independent of $\nu_{1}$.

In a similar manner we estimate the third term on the right-hand side of (1.3)

$$
\left|\int_{0}^{t} \overrightarrow{f_{2}}\left(\vec{x}(s), \nu_{2} s\right) d s\right| \leqslant \frac{d_{2}}{\nu_{2}}
$$

where $d_{2}$ is the constant independent of $\nu_{2}$. Finally, from (1.3) we have

$$
\left|\vec{u}\left(t, \nu_{1}, \nu_{2}, \vec{x}_{0}\right)\right| \leqslant L \int_{0}^{t}\left|\vec{u}\left(s, \nu_{1}, \nu_{2}, \vec{x}_{0}\right)\right| d s+\frac{d_{1}}{\nu_{1}}+\frac{d_{2}}{\nu_{2}} .
$$

Then, using the Gronwall-Bellman lemma, we obtain

$$
\left|\vec{u}\left(t, \nu_{1}, \nu_{2}, \vec{x}_{0}\right)\right| \leqslant\left(\frac{d_{1}}{\nu_{1}}+\frac{d_{2}}{\nu_{2}}\right) e^{L t} \leqslant \frac{d_{1} e^{L a}}{\nu_{1}}+\frac{d_{2} e^{L a}}{\nu_{2}}=\frac{c_{1}}{\nu_{1}}+\frac{c_{2}}{\nu_{2}}
$$

for any $t \in[0, a]$. This proves the statement.

REMARK 1 . If the system is a single-frequency system, i.e., $\vec{f}_{2} \equiv 0$ or $\nu_{2}=\nu_{1}$, then Statement 1 gives the same estimate as the Krylov-Bogolyubov averaging theorem.

Remark 2. Consider the system of equations

$$
\dot{\vec{x}}=\vec{g}(\vec{x}, t)+\vec{f}_{1}\left(\vec{x}, t, \nu_{1} t\right)+\vec{f}_{2}\left(\vec{x}, t, \nu_{2} t\right), \quad \vec{x} \in \mathbb{R}^{n}, \quad \nu_{1}>0, \nu_{2}>0,
$$

where $\vec{g}(\vec{x}, t), \vec{f}_{1}(\vec{x}, t, \tau), \vec{f}_{2}(\vec{x}, t, \tau) \in \mathbb{R}^{n}$ are functions that are continuously differentiable with respect to the variables $\vec{x} \in \mathbb{R}^{n}, t, \tau \in \mathbb{R}$ and the functions $\vec{f}_{1}(\vec{x}, t, \tau), \vec{f}_{2}(\vec{x}, t, \tau)$ are periodic in $\tau$ with period 1 , independent of $\nu_{1}, \nu_{2}$, and their average on a period over $\tau$ is zero. This system of equations can be autonomized by replacing in the functions $\vec{g}(\vec{x}, t), \vec{f}_{1}(\vec{x}, t, \tau)$ and $\vec{f}_{2}(\vec{x}, t, \tau)$ time $t$ by an additional variable $x_{n+1}$ and by setting $\dot{x}_{n+1}=1$. As a result, the system (1.6) is brought to the form (1.1)

$$
\dot{\vec{x}}=\overrightarrow{\vec{g}}(\overrightarrow{\vec{x}})+\overrightarrow{\widetilde{f}}_{1}\left(\overrightarrow{\vec{x}}, \nu_{1} t\right)+\overrightarrow{\widetilde{f}}_{2}\left(\overrightarrow{\vec{x}}, \nu_{2} t\right)
$$

where

$$
\overrightarrow{\widetilde{x}}=\left(\begin{array}{c}
\vec{x} \\
x_{n+1}
\end{array}\right), \quad \overrightarrow{\widetilde{g}}=\left(\begin{array}{c}
\vec{g} \\
1
\end{array}\right), \quad \overrightarrow{\widetilde{f}}_{1}=\left(\begin{array}{c}
\vec{f}_{1} \\
0
\end{array}\right), \quad \overrightarrow{\widetilde{f}}_{2}=\left(\begin{array}{c}
\vec{f}_{2} \\
0
\end{array}\right) .
$$

Thus, Statement 1 holds for systems (1.6) too. 
Statement 1 is generalized to the case of three and more frequencies. Consider the system of ordinary differential equations

$$
\dot{\vec{x}}=\vec{g}(\vec{x})+\vec{f}_{1}\left(\vec{x}, \nu_{1} t\right)+\ldots+\vec{f}_{k}\left(\vec{x}, \nu_{k} t\right), \quad \vec{x} \in \mathbb{R}^{n}, \quad \nu_{i}>0, i=1, \ldots, k .
$$

Here the functions $\vec{g}(\vec{x}), \vec{f}_{i}(\vec{x}, \tau)$ are continuously differentiable with respect to the variables $\vec{x} \in \mathbb{R}^{n}, \tau \in \mathbb{R}$, the functions $\vec{f}_{i}(\vec{x}, \tau)$ are periodic in $\tau$ with period 1 , independent of $\nu_{1}, \ldots, \nu_{k}$, and their average on a period is zero.

Statement 2. Suppose that for some $a>0$ and any $\nu_{i}>0(i=1, \ldots, k)$ the solutions $\vec{x}\left(t, \nu_{1}, \ldots, \nu_{k}, \vec{x}_{0}\right)$ and $\vec{z}\left(t, \vec{x}_{0}\right)$ to systems $(1.7),(1.2)$ with initial conditions $\vec{x}\left(0, \nu_{1}, \ldots, \nu_{k}, \vec{x}_{0}\right)=$ $=\vec{x}_{0}$ and $\vec{z}\left(0, \vec{x}_{0}\right)=\vec{x}_{0}$, where $\vec{x}_{0}$ lies in some bounded region $U \subset \mathbb{R}^{n}$, exist on the interval $0 \leqslant t \leqslant a$ and lie in some bounded region $V \subset \mathbb{R}^{n}$. Then one can find constants $c_{1}, \ldots, c_{k}>0$ such that for all $\vec{x}_{0} \in U$ one has

$$
\left|\vec{x}\left(t, \nu_{1}, \ldots, \nu_{k}, \vec{x}_{0}\right)-\vec{z}\left(t, \vec{x}_{0}\right)\right| \leqslant \frac{c_{1}}{\nu_{1}}+\ldots+\frac{c_{k}}{\nu_{k}} \quad \text { with } \quad 0 \leqslant t \leqslant a .
$$

\section{Step-by-step averaging in systems of general form}

Consider the system of ordinary differential equations

$$
\dot{\vec{x}}=\vec{f}\left(\vec{x}, \nu_{1} t, \nu_{2} t\right), \quad \vec{x} \in \mathbb{R}^{n}, \quad \nu_{1}>0, \nu_{2}>0,
$$

where $\vec{f}\left(\vec{x}, \tau_{1}, \tau_{2}\right)$ is a function that is continuously differentiable with respect to all variables, periodic in the variables $\tau_{1}, \tau_{2}$ with period 1 , and independent of $\nu_{1}, \nu_{2}$. Averaging the right-hand side of the system (2.1) over $\tau_{1}$ and $\tau_{2}$, we write the averaged system

$$
\dot{\vec{z}}=\vec{g}(\vec{z}), \quad \vec{g}(\vec{z})=\int_{0}^{1} \int_{0}^{1} \vec{f}\left(\vec{x}, \tau_{1}, \tau_{2}\right) d \tau_{1} d \tau_{2} .
$$

Statement 3. Suppose that for some $a>0$ and any $\nu_{1}, \nu_{2}>0$ the solutions $\vec{x}\left(t, \nu_{1}, \nu_{2}, \vec{x}_{0}\right)$ and $\vec{z}\left(t, \vec{x}_{0}\right)$ to systems $(2.1),(2.2)$ with initial conditions $\vec{x}\left(0, \nu_{1}, \nu_{2}, \vec{x}_{0}\right)=\vec{x}_{0}$ and $\vec{z}\left(0, \vec{x}_{0}\right)=\vec{x}_{0}$, where $\vec{x}_{0}$ lies in some bounded region $U \subset \mathbb{R}^{n}$, exist on the interval $0 \leqslant t \leqslant a$ and lie in some bounded region $V \subset \mathbb{R}^{n}$. Then one can find constants $c_{1}, c_{2}, c_{3}>0$ such that for all $\vec{x}_{0} \in U$ the estimate of the deviation of the solution $\vec{z}\left(t, \vec{x}_{0}\right)$ to the averaged system from the solution $\vec{x}\left(t, \nu_{1}, \nu_{2}, \vec{x}_{0}\right)$ to the initial system has the form

$$
\left|\vec{x}\left(t, \nu_{1}, \nu_{2}, \vec{x}_{0}\right)-\vec{z}\left(t, \vec{x}_{0}\right)\right| \leqslant \frac{c_{1}}{\nu_{1}}+\frac{c_{2} \nu_{2}}{\nu_{1}}+\frac{c_{3}}{\nu_{2}} \quad \text { with } \quad 0 \leqslant t \leqslant a .
$$

Proof. a) Averaging the function $\vec{f}\left(\vec{x}, \tau_{1}, \tau_{2}\right)$ over the variable $\tau_{1}$

$$
\int_{0}^{1} f\left(\vec{x}, \tau_{1}, \tau_{2}\right) d \tau_{1}=\vec{g}_{1}\left(\vec{x}, \tau_{2}\right)
$$

we write the system $(2.1)$ as

$$
\dot{\vec{x}}=\vec{g}_{1}\left(\vec{x}, \nu_{2} t\right)+\vec{f}_{1}\left(\vec{x}, \nu_{1} t, \nu_{2} t\right),
$$

where the function $\vec{f}_{1}\left(\vec{x}, \tau_{1}, \tau_{2}\right)=\vec{f}\left(\vec{x}, \tau_{1}, \tau_{2}\right)-\vec{g}_{1}\left(\vec{x}, \tau_{2}\right)$ has a zero average over $\tau_{1}$. 
Consider the system

$$
\dot{\vec{w}}=\vec{g}_{1}\left(\vec{w}, \nu_{2} t\right) .
$$

Let $\vec{w}\left(t, \nu_{2}, \vec{x}_{0}\right)$ be a solution to the system $(2.5)$ on the interval $0 \leqslant t \leqslant a$ with initial conditions $\vec{w}\left(0, \nu_{2}, \vec{x}_{0}\right)=\vec{x}_{0}$. We show that

$$
\left|\vec{x}\left(t, \nu_{1}, \nu_{2}, \vec{x}_{0}\right)-\vec{w}\left(t, \nu_{2}, \vec{x}_{0}\right)\right| \leqslant \frac{c_{1}}{\nu_{1}}+\frac{c_{2} \nu_{2}}{\nu_{1}} \quad \text { with } \quad 0 \leqslant t \leqslant a .
$$

To this end, introducing the additional variable $x_{n+1}$, which changes in accordance with the equation $\dot{x}_{n+1}=\nu_{2}$, we reduce the system (2.4) to the form

$$
\dot{\overrightarrow{\widetilde{x}}}=\overrightarrow{\widetilde{g}}_{1}(\overrightarrow{\widetilde{x}})+\overrightarrow{\widetilde{f}}_{1}\left(\overrightarrow{\widetilde{x}}, \nu_{1} t\right)
$$

where

$$
\overrightarrow{\widetilde{x}}=\left(\begin{array}{c}
\vec{x} \\
x_{n+1}
\end{array}\right), \quad \overrightarrow{\widetilde{g}}_{1}(\overrightarrow{\vec{x}})=\left(\begin{array}{c}
\vec{g}_{1}\left(\vec{x}, x_{n+1}\right) \\
\nu_{2}
\end{array}\right), \quad \overrightarrow{\widetilde{f}}_{1}\left(\overrightarrow{\widetilde{x}}, \tau_{1}\right)=\left(\begin{array}{c}
\vec{f}_{1}\left(\vec{x}, \tau_{1}, x_{n+1}\right) \\
0
\end{array}\right)
$$

and write its averaged system

$$
\dot{\overrightarrow{\widetilde{w}}}=\overrightarrow{\widetilde{g}}_{1}(\overrightarrow{\widetilde{w}}), \quad \overrightarrow{\widetilde{w}} \in \mathbb{R}^{n+1}
$$

Let us estimate the difference $\overrightarrow{\widetilde{x}}\left(t, \nu_{1}, \overrightarrow{\widetilde{x}}_{0}\right)-\overrightarrow{\widetilde{w}}\left(t, \overrightarrow{\widetilde{x}}_{0}\right)$ of the solutions to the systems $(2.7)$ and $(2.8)$ on the interval $0 \leqslant t \leqslant a$. We have

$$
\left|\overrightarrow{\widetilde{x}}\left(t, \nu_{1}, \overrightarrow{\widetilde{x}}_{0}\right)-\overrightarrow{\widetilde{w}}\left(t, \overrightarrow{\widetilde{x}}_{0}\right)\right|=\left|\overrightarrow{\widetilde{u}}\left(t, \nu_{1}, \overrightarrow{\widetilde{x}}_{0}\right)\right| \leqslant\left|\int_{0}^{t}\left(\overrightarrow{\widetilde{g}}_{1}(\overrightarrow{\widetilde{x}}(s))-\overrightarrow{\widetilde{g}}_{1}(\overrightarrow{\widetilde{w}}(s))\right) d s\right|+\left|\int_{0}^{t} \overrightarrow{\widetilde{f}}_{1}\left(\overrightarrow{\widetilde{x}}(s), \nu_{1} s\right) d s\right|
$$

Since the function $\vec{f}\left(\vec{x}, \tau_{1}, \tau_{2}\right)$ is continuously differentiable with respect to all variables and is periodic in the variables $\tau_{1}, \tau_{2}$, it follows that in the region $V \subset \mathbb{R}^{n}$ for any $\tau_{1}, \nu_{1}, \nu_{2}$ the functions $\vec{g}_{1}, \vec{f}_{1}$ are bounded by some constant $K$ :

$$
\left|\vec{g}_{1}\left(\vec{x}, x_{n+1}\right)\right| \leqslant K, \quad\left|\vec{f}_{1}\left(\vec{x}, \tau_{1}, x_{n+1}\right)\right| \leqslant K
$$

and satisfy the Lipschitz condition in $\overrightarrow{\widetilde{x}}$ with some constant $L$ :

$$
\left|\vec{g}_{1}\left(\vec{x}, x_{n+1}\right)-\vec{g}_{1}\left(\vec{x}^{\prime}, x_{n+1}^{\prime}\right)\right| \leqslant L\left|\overrightarrow{\vec{x}}-\overrightarrow{\vec{x}}^{\prime}\right|, \quad\left|\vec{f}_{1}\left(\vec{x}, \tau_{1}, x_{n+1}\right)-\vec{f}_{1}\left(\vec{x}^{\prime}, \tau_{1}, x_{n+1}^{\prime}\right)\right| \leqslant L\left|\overrightarrow{\vec{x}}-\overrightarrow{\vec{x}}^{\prime}\right|
$$

Following the algorithm for proving Statement 1, we obtain

$$
\begin{gathered}
\left|\int_{0}^{t}\left(\overrightarrow{\widetilde{g}}_{1}(\overrightarrow{\vec{x}}(s))-\overrightarrow{\vec{g}}_{1}(\overrightarrow{\vec{w}}(s))\right) d s\right| \leqslant L \int_{0}^{t}\left|\overrightarrow{\vec{u}}\left(s, \nu_{1}, \overrightarrow{\widetilde{x}}_{0}\right)\right| d s, \quad\left|\dot{\vec{x}}\left(t, \nu_{1}, \overrightarrow{\vec{x}}_{0}\right)\right| \leqslant 2 K+\nu_{2}, \\
\left|\int_{0}^{t} \overrightarrow{\widetilde{f}}_{1}\left(\overrightarrow{\widetilde{x}}(s), \nu_{1} s\right) d s\right| \leqslant\left|\int_{0}^{t_{m}} \overrightarrow{\widetilde{f}}_{1}\left(\overrightarrow{\widetilde{x}}(s), \nu_{1} s\right) d s\right|+\left|\int_{t_{m}}^{t} \overrightarrow{\widetilde{f}}_{1}\left(\overrightarrow{\widetilde{x}}(s), \nu_{1} s\right) d s\right| \leqslant \\
\leqslant \frac{a L\left(2 K+\nu_{2}\right)}{2 \nu_{1}}+\frac{K}{\nu_{1}}=\frac{d_{1}}{\nu_{1}}+\frac{d_{2} \nu_{2}}{\nu_{1}}
\end{gathered}
$$


where $d_{1}, d_{2}>0$ are constants independent of $\nu_{1}, \nu_{2}$. Then

$$
\left|\overrightarrow{\widetilde{u}}\left(t, \nu_{1}, \overrightarrow{\widetilde{x}}_{0}\right)\right| \leqslant\left(\frac{d_{1}}{\nu_{1}}+\frac{d_{2} \nu_{2}}{\nu_{1}}\right) e^{L a}=\frac{c_{1}}{\nu_{1}}+\frac{c_{2} \nu_{2}}{\nu_{1}} \quad \text { with } \quad 0 \leqslant t \leqslant a
$$

and hence inequality (2.6) holds.

We note that

$$
c_{2}=\frac{L a}{2} e^{L a} .
$$

This equality is used below in the proof of Statement 5 .

b) Averaging the function $\vec{g}_{1}\left(\vec{x}, \tau_{2}\right)$ over $\tau_{2}$

$$
\int_{0}^{1} \vec{g}_{1}\left(\vec{x}, \tau_{2}\right) d \tau_{2}=\int_{0}^{1} \int_{0}^{1} \vec{f}\left(\vec{x}, \tau_{1}, \tau_{2}\right) d \tau_{1} d \tau_{2}=\vec{g}(\vec{x}),
$$

we obtain for the system (2.5) an averaged system that coincides with the system (2.2). In accordance with Statement 1 , the estimate of the deviation of the solution $\vec{z}\left(t, \vec{x}_{0}\right)$ to the system $(2.2)$ from the solution $\vec{w}\left(t, \nu_{2}, \vec{x}_{0}\right)$ to the system (2.5) has the form

$$
\left|\vec{w}\left(t, \nu_{2}, \vec{x}_{0}\right)-\vec{z}\left(t, \vec{x}_{0}\right)\right| \leqslant \frac{c_{3}}{\nu_{2}} \quad \text { with } \quad 0 \leqslant t \leqslant a .
$$

Then, using (2.6) and (2.10) with $0 \leqslant t \leqslant a$, we obtain

$$
\begin{gathered}
\left|\vec{x}\left(t, \nu_{1}, \nu_{2}, \vec{x}_{0}\right)-\vec{z}\left(t, \vec{x}_{0}\right)\right| \leqslant\left|\vec{x}\left(t, \nu_{1}, \nu_{2}, \vec{x}_{0}\right)-\vec{w}\left(t, \nu_{2}, \vec{x}_{0}\right)\right|+\left|\vec{w}\left(t, \nu_{2}, \vec{x}_{0}\right)-\vec{z}\left(t, \vec{x}_{0}\right)\right| \leqslant \\
\leqslant \frac{c_{1}}{\nu_{1}}+\frac{c_{2} \nu_{2}}{\nu_{1}}+\frac{c_{3}}{\nu_{2}} .
\end{gathered}
$$

This completes the proof.

If one changes the order of averaging and averages first over $\tau_{2}$ and then over $\tau_{1}$, the estimate of the deviation of the solution to the averaged system from the solution to the initial system will have the form

$$
\left|\vec{x}\left(t, \nu_{1}, \nu_{2}, \vec{x}_{0}\right)-\vec{z}\left(t, \vec{x}_{0}\right)\right| \leqslant \frac{c_{1}^{*}}{\nu_{1}}+\frac{c_{2}^{*} \nu_{1}}{\nu_{2}}+\frac{c_{3}^{*}}{\nu_{2}} \quad \text { with } \quad 0 \leqslant t \leqslant a,
$$

where $c_{1}^{*}, c_{2}^{*}, c_{3}^{*}>0$ are some constants independent of $\nu_{1}, \nu_{2}$. From the two estimates, (2.3) and (2.11), one should choose the best. For example, for the case of large frequencies, if $\nu_{1} \gg \nu_{2} \gg 1$, the best estimate is (2.3), from which we obtain

$$
\left|\vec{x}\left(t, \nu_{1}, \nu_{2}, \vec{x}_{0}\right)-\vec{z}\left(t, \vec{x}_{0}\right)\right|<c\left(\frac{1}{\nu_{2}}+\frac{\nu_{2}}{\nu_{1}}\right) \quad \text { with } \quad 0 \leqslant t \leqslant a,
$$

where $c>0$ is some constant independent of $\nu_{1}, \nu_{2}$.

Statement 3 is generalized to the case of three and more frequencies. Consider the system of ordinary differential equations

$$
\dot{\vec{x}}=\vec{f}\left(\vec{x}, \nu_{1} t, \nu_{2} t, \ldots, \nu_{k} t\right), \quad \vec{x} \in \mathbb{R}^{n},
$$


where $\vec{f}\left(\vec{x}, \tau_{1}, \tau_{2}, \ldots, \tau_{k}\right)$ is a function that is continuously differentiable with respect to all variables, periodic in the variables $\tau_{1}, \ldots, \tau_{k}$ with period 1 , and independent of $\nu_{1}, \ldots, \nu_{k}$. We assume that the frequencies $\nu_{i}$ have been renumbered in decreasing order

$$
\nu_{1}>\nu_{2}>\ldots>\nu_{k}>0
$$

Let us average the right-hand side of the system (2.12) over $\tau_{1}, \ldots, \tau_{k}$ and write the averaged system

$$
\dot{\vec{z}}=\vec{g}(\vec{z}), \quad \text { where } \quad \vec{g}(\vec{z})=\int_{0}^{1} \ldots \int_{0}^{1} \vec{f}\left(\vec{z}, \tau_{1}, \ldots, \tau_{k}\right) d \tau_{1} \ldots d \tau_{k} .
$$

Statement 4. Suppose that for some $a>0$ and any $\nu_{1}, \ldots, \nu_{k}>0$ the solutions $\vec{x}\left(t, \nu_{1}, \ldots, \nu_{k}, \vec{x}_{0}\right)$ and $\vec{z}\left(t, \vec{x}_{0}\right)$ to the systems (2.12), (2.13) with initial conditions $\vec{x}\left(0, \nu_{1}, \ldots, \nu_{k}, \vec{x}_{0}\right)=\vec{x}_{0}$ and $\vec{z}\left(0, \vec{x}_{0}\right)=\vec{x}_{0}$, where $\vec{x}_{0}$ lies in some bounded region $U \subset \mathbb{R}^{n}$, exist on the interval $0 \leqslant t \leqslant a$ and lie in some bounded region $V \subset \mathbb{R}^{n}$. Then one can find constants $c_{1}, \ldots, c_{k}, c_{1}^{*}, \ldots, c_{k-1}^{*}>0$ such that for all $\vec{x}_{0} \in U$ with $0 \leqslant t \leqslant a$ one has

$$
\left|\vec{x}\left(t, \nu_{1}, \ldots, \nu_{k}, \vec{x}_{0}\right)-\vec{z}\left(t, \vec{x}_{0}\right)\right| \leqslant \frac{c_{1}}{\nu_{1}}+\frac{c_{2}}{\nu_{2}}+\ldots+\frac{c_{k}}{\nu_{k}}+\frac{c_{1}^{*} \nu_{2}}{\nu_{1}}+\frac{c_{2}^{*} \nu_{3}}{\nu_{2}}+\ldots+\frac{c_{k-1}^{*} \nu_{k}}{\nu_{k-1}} .
$$

Proof. Averaging step by step the function $\vec{f}\left(\vec{x}, \tau_{1}, \tau_{2}, \ldots, \tau_{k}\right)$ over the variables $\tau_{1}, \tau_{2}, \ldots, \tau_{k-1}$, we obtain the averaged systems

$$
\dot{\vec{z}}_{i}=\vec{g}_{i}\left(\vec{z}_{i}, \nu_{i+1} t, \ldots, \nu_{k} t\right), \quad i=1, \ldots, k-1,
$$

where $\vec{g}_{i}\left(\vec{x}, \tau_{i+1}, \ldots, \tau_{k}\right)=\int_{0}^{1} \ldots \int_{0}^{1} \vec{f}\left(\vec{x}, \tau_{1}, \ldots, \tau_{k}\right) d \tau_{1} \ldots d \tau_{i} . \quad$ Let $\quad \vec{z}_{i}\left(t, \nu_{i+1}, \ldots, \nu_{k}, \vec{x}_{0}\right)$ $(i=1, \ldots, k-1)$ be a solution to the system (2.15) on the interval $0 \leqslant t \leqslant a$ with initial conditions $\vec{z}_{i}\left(0, \nu_{i+1}, \ldots, \nu_{k}, \vec{x}_{0}\right)=\vec{x}_{0}$. For $0 \leqslant t \leqslant a$, in accordance with the estimates $(2.6)$ and (2.10) obtained in the proof of Statement 3, we have

$$
\begin{gathered}
\left|\vec{x}\left(t, \nu_{1}, \ldots, \nu_{k}, \vec{x}_{0}\right)-\vec{z}_{1}\left(t, \nu_{2}, \ldots, \nu_{k}, \vec{x}_{0}\right)\right| \leqslant \frac{c_{1}}{\nu_{1}}+\frac{c_{1}^{*} \nu_{2}}{\nu_{1}} \\
\left|\vec{z}_{i-1}\left(t, \nu_{i}, \ldots, \nu_{k}, \vec{x}_{0}\right)-\vec{z}_{i}\left(t, \nu_{i+1}, \ldots, \nu_{k}, \vec{x}_{0}\right)\right| \leqslant \frac{c_{i}}{\nu_{i}}+\frac{c_{i}^{*} \nu_{i+1}}{\nu_{i}}, \quad i=2, \ldots, k-1 \\
\left|\vec{z}_{k-1}\left(t, \nu_{k}, \vec{x}_{0}\right)-\vec{z}\left(t, \vec{x}_{0}\right)\right| \leqslant \frac{c_{k}}{\nu_{k}} .
\end{gathered}
$$

Using these inequalities and the inequality

$$
\begin{aligned}
& \left|\vec{x}\left(t, \nu_{1}, \ldots, \nu_{k}, \vec{x}_{0}\right)-\vec{z}\left(t, \vec{x}_{0}\right)\right| \leqslant\left|\vec{x}\left(t, \nu_{1}, \ldots, \nu_{k}, \vec{x}_{0}\right)-\vec{z}_{1}\left(t, \nu_{2}, \ldots, \nu_{k}, \vec{x}_{0}\right)\right|+ \\
+ & \left|\vec{z}_{1}\left(t, \nu_{2}, \ldots, \nu_{k}, \vec{x}_{0}\right)-\vec{z}_{2}\left(t, \nu_{3}, \ldots, \nu_{k}, \vec{x}_{0}\right)\right|+\ldots+\left|\vec{z}_{k-1}\left(t, \nu_{k}, \vec{x}_{0}\right)-\vec{z}\left(t, \vec{x}_{0}\right)\right|,
\end{aligned}
$$

we obtain the estimate (2.14).

Taking the inequalities $\nu_{1}>\nu_{2}>\ldots>\nu_{k}$ into account, we can write the expression (2.14) for estimating the deviation of the solution $\vec{z}\left(t, \vec{x}_{0}\right)$ to the averaged system from the solution $\vec{x}\left(t, \nu_{1}, \ldots, \nu_{k}, \vec{x}_{0}\right)$ to the initial system with $0 \leqslant t \leqslant a$ as follows:

$$
\left|\vec{x}\left(t, \nu_{1}, \ldots, \nu_{k}, \vec{x}_{0}\right)-\vec{z}\left(t, \vec{x}_{0}\right)\right|<c\left(\frac{\nu_{2}}{\nu_{1}}+\frac{\nu_{3}}{\nu_{2}}+\ldots+\frac{\nu_{k}}{\nu_{k-1}}+\frac{1}{\nu_{k}}\right) .
$$




\section{Examples}

In this section, we consider a pendulum with a vibrating point of suspension. The problem of the motion of pendulum-type systems with vibrating elements is a classical one [10-12]. Foremost among studies concerned with such systems are [13-19].

\subsection{A mathematical pendulum with a vibrating point of suspension in a rotating coordinate system}

Consider a mathematical pendulum of length $l$ whose plane undergoes rotational oscillations about a fixed vertical axis $O z$ and whose point of suspension oscillates along this axis. The change in the coordinate of the point of suspension on the axis $O z$ and in the angle $\theta$ of rotation of the pendulum's plane about the axis $O z$ is given by the relations

$$
\dot{z}_{0}(t)=a h_{1}\left(\nu_{1} t\right), \quad \dot{\theta}(t)=b h_{2}\left(\nu_{2} t\right) .
$$

Here $a, b>0$ are constants, $h_{1}(\tau), h_{2}(\tau)$ are continuous periodic functions with zero average value which are different from a constant, and $\nu_{1}, \nu_{2}>0$ are oscillation frequencies.

Let $\varphi$ denote the angle of deviation of the pendulum from the descending vertical and let us write the Hamiltonian function of the system. In the fixed coordinate system Oxyz the coordinates of the pendulum are

$$
x=l \sin \varphi \cos \theta, \quad y=l \sin \varphi \sin \theta, \quad z=z_{0}-l \cos \varphi,
$$

therefore, its squared velocity is

$$
v^{2}=l^{2} \dot{\varphi}^{2}+l^{2} \dot{\theta}^{2} \sin ^{2} \varphi+2 l \dot{\varphi} \dot{z}_{0} \sin \varphi+\dot{z}_{0}^{2} .
$$

The Lagrangian function of the system (up to calibration) has the form

$$
L(\varphi, \dot{\varphi}, t)=\frac{l \dot{\varphi}^{2}}{2}+\dot{\varphi} \dot{z}_{0} \sin \varphi+\frac{l \dot{\theta}^{2} \sin ^{2} \varphi}{2}+g \cos \varphi .
$$

Introducing the generalized momentum

$$
p=\frac{\partial L}{\partial \dot{\varphi}}=l \dot{\varphi}+\dot{z}_{0} \sin \varphi
$$

we write the Hamiltonian function of the system

$$
H(\varphi, p, t)=\frac{\left(p-\dot{z}_{0} \sin \varphi\right)^{2}}{2 l}-\frac{l \dot{\theta}^{2} \sin ^{2} \varphi}{2}-g \cos \varphi
$$

where $\dot{z}_{0}(t), \dot{\theta}(t)$ are given by (3.1). The Hamiltonian function is represented as a sum of the Hamiltonian function of the system unperturbed by oscillations, and two time-periodic functions with frequencies $\nu_{1}, \nu_{2}$ :

$$
\begin{gathered}
H(\varphi, p, t)=H_{0}(\varphi, p)+H_{1}\left(\varphi, p, \tau_{1}\right)+H_{2}\left(\varphi, \tau_{2}\right), \quad H_{0}(\varphi, p)=\frac{p^{2}}{2 l}-g \cos \varphi \\
H_{1}\left(\varphi, p, \tau_{1}\right)=-\frac{a p \sin \varphi h_{1}\left(\tau_{1}\right)}{l}+\frac{a^{2} \sin ^{2} \varphi h_{1}^{2}\left(\tau_{1}\right)}{2 l}, \quad \tau_{1}=\nu_{1} t \\
H_{2}\left(\varphi, \tau_{2}\right)=-\frac{l b^{2} \sin ^{2} \varphi h_{2}^{2}\left(\tau_{2}\right)}{2}, \quad \tau_{2}=\nu_{2} t
\end{gathered}
$$


Thus, one can use Statement 1 about averaging in a two-frequency system. The right-hand side of the Hamilton equations is given by partial derivatives of the Hamiltonian function in the canonical variables $\varphi, p$. Therefore, the averaged Hamilton equations are also Hamilton equations with the Hamiltonian (of the initial system) averaged over the variables $\tau_{1}, \tau_{2}$. The average values of the functions $H_{1}\left(\varphi, p, \tau_{1}\right)$ and $H_{2}\left(\varphi, \tau_{2}\right)$ over the variables $\tau_{1}$ and $\tau_{2}$ are, respectively,

$$
\bar{H}_{1}(\varphi)=\frac{1}{s_{1}} \int_{0}^{s_{1}} H_{1}\left(\varphi, p, \tau_{1}\right) d \tau_{1}=\frac{a^{2} \sin ^{2} \varphi \overline{h_{1}^{2}}}{2 l}, \quad \bar{H}_{2}(\varphi)=\frac{1}{s_{2}} \int_{0}^{s_{2}} H_{2}\left(\varphi, \tau_{2}\right) d \tau_{2}=-\frac{l b^{2} \sin ^{2} \varphi \overline{h_{2}^{2}}}{2} .
$$

Here $s_{i}$ is the period of the function $h_{i}(\tau)$ and $\overline{h_{i}^{2}}=\frac{1}{s_{i}} \int_{0}^{s_{i}} h_{i}^{2}(\tau) d \tau, i=1,2$. Then the Hamiltonian of the averaged system is

$$
\bar{H}(\varphi, p)=H_{0}(\varphi, p)+\bar{H}_{1}(\varphi)+\bar{H}_{2}(\varphi)=\frac{p^{2}}{2 l}-g \cos \varphi+\frac{a^{2} \overline{h_{1}^{2}}-l^{2} b^{2} \overline{h_{2}^{2}}}{2 l} \sin ^{2} \varphi .
$$

Let $\varphi\left(t, \nu_{1}, \nu_{2}\right), p\left(t, \nu_{1}, \nu_{2}\right)$ be a solution to the Hamilton equations with Hamiltonian (3.2) on the interval $0 \leqslant t \leqslant a(a>0)$, and let $\varphi^{*}(t), p^{*}(t)$ be a solution with the same initial conditions to the averaged Hamilton equations with Hamiltonian (3.3) on the interval $0 \leqslant t \leqslant a$. In accordance with Statement 1, the estimate of the deviation of the solution to the averaged system from the solution to the initial system has the form

$$
\left|\varphi\left(t, \nu_{1}, \nu_{2}\right)-\varphi^{*}(t)\right|+\left|p\left(t, \nu_{1}, \nu_{2}\right)-p^{*}(t)\right| \leqslant \frac{c_{1}}{\nu_{1}}+\frac{c_{2}}{\nu_{2}} \quad \text { with } \quad 0 \leqslant t \leqslant a,
$$

where $c_{1}, c_{2}>0$ are some constants independent of $\nu_{1}, \nu_{2}$. Thus, the solutions of the initial system tend uniformly in $t$ to solutions of the averaged system if the oscillation frequencies $\nu_{1}, \nu_{2}$ tend to infinity.

Let us investigate the stability of the equilibrium points of the averaged system. According to $(3.3)$, the potential energy $V^{*}(\varphi)$ of the averaged system is

$$
V^{*}(\varphi)=-g \cos \varphi+\frac{a^{2} \overline{h_{1}^{2}}-l^{2} b^{2} \overline{h_{2}^{2}}}{2 l} \sin ^{2} \varphi .
$$

The equilibrium points of the averaged system are found from the equation

$$
\frac{d V^{*}}{d \varphi}=\sin \varphi\left(g+\frac{k}{l} \cos \varphi\right)=0
$$

where $k=a^{2} \overline{h_{1}^{2}}-l^{2} b^{2} \overline{h_{2}^{2}}$. The system always has two equilibrium points: $\varphi_{1}=0$ (the lower one) and $\varphi_{2}=\pi$ (the upper one). When $|k|>g l$, the system has two more equilibrium points (lateral equilibria)

$$
\varphi_{3,4}= \pm \arccos \left(-\frac{g l}{k}\right)
$$

The lower equilibrium point is stable when $\left.\frac{d^{2} V^{*}}{d \varphi^{2}}\right|_{\varphi=0}=g+\frac{k}{l}>0$, i.e., when $k>-g l$. The upper equilibrium point is stable when $\left.\frac{d^{2} V^{*}}{d \varphi^{2}}\right|_{\varphi=\pi}=-g+\frac{k}{l}>0$, i.e., when $k>g l$. The lateral 
equilibrium points are stable when $\left.\frac{d^{2} V^{*}}{d \varphi^{2}}\right|_{\varphi=\varphi_{3,4}}=-\left.\frac{k}{l} \sin ^{2} \varphi\right|_{\varphi=\varphi_{3,4}}>0$, i.e., when $k<-g l$. When $k=-g l$, the lower equilibrium point is degenerately stable. When $k=g l$, the upper equilibrium point is degenerately unstable.

Thus, we obtain:

When $k<-g l$, the averaged system has four different equilibria: a lower, an upper and two lateral equilibria located below the point of suspension of the pendulum, the lower and upper equilibria being unstable and the lateral ones stable.

When $-g l \leqslant k \leqslant g l$, the averaged system has only two different equilibria: a stable lower and an unstable upper equilibrium.

When $k>g l$, the averaged system has four different equilibria: a lower, an upper and two lateral equilibria located above the point of suspension of the pendulum, the lower and the upper equilibria being stable, and the lateral ones unstable.

In contrast to the classical problem of a pendulum with a point of suspension vibrating along a vertical $(b=0)$, in this problem the upper equilibrium point of the averaged system becomes stable at a large value of the amplitude of velocity of vertical oscillations: $a^{2}>\frac{g l+l^{2} b^{2} \overline{h_{2}^{2}}}{\overline{h_{1}^{2}}}$. Also, a situation is possible where the lower equilibrium point becomes unstable.

\subsection{A mathematical pendulum with a vibrating point of suspension}

Consider a mathematical pendulum of length $l$ which moves in a fixed vertical plane $O x z$ and whose point of suspension undergoes oscillations such that the velocity components of the point of suspension in the fixed coordinate system $O x z$ are

$$
\dot{x}_{0}(t)=b h_{2}\left(\nu_{2} t\right), \quad \dot{z}_{0}(t)=a h_{1}\left(\nu_{1} t\right),
$$

where $a, b>0$ are constants, $h_{1}(\tau), h_{2}(\tau)$ are continuous periodic functions that are different from a constant and have zero mean value, and $\nu_{1}, \nu_{2}>0$ are oscillation frequencies. The dynamics of such a system was studied, for example, in [20, 21].

Letting $\varphi$ denote the angle of deviation of the pendulum from the descending vertical, we set up the Hamiltonian function of the system. In the fixed coordinate system $O x z$ the coordinates of the pendulum are

$$
x=x_{0}+l \sin \varphi, \quad z=z_{0}-l \cos \varphi,
$$

and hence its squared velocity is

$$
v^{2}=l^{2} \dot{\varphi}^{2}+2 l \dot{\varphi} \dot{x}_{0} \cos \varphi+2 l \dot{\varphi} \dot{z}_{0} \sin \varphi+\dot{x}_{0}^{2}+\dot{z}_{0}^{2}
$$

Then the Lagrange function (up to calibration), the generalized momentum and the Hamiltonian function of the system have the form

$$
\begin{gathered}
L(\varphi, \dot{\varphi}, t)=\frac{l \dot{\varphi}^{2}}{2}+\dot{\varphi} \dot{x}_{0} \cos \varphi+\dot{\varphi} \dot{z}_{0} \sin \varphi+g \cos \varphi \\
p=\frac{\partial L}{\partial \dot{\varphi}}=l \dot{\varphi}+\dot{x}_{0} \cos \varphi+\dot{z}_{0} \sin \varphi \\
H(\varphi, p, t)=\frac{\left(p-\dot{x}_{0} \cos \varphi-\dot{z}_{0} \sin \varphi\right)^{2}}{2 l}-g \cos \varphi,
\end{gathered}
$$


where $\dot{x}_{0}(t), \dot{z}_{0}(t)$ are given by (3.5). The Hamiltonian function is represented as a sum of the Hamiltonian function of the system unperturbed by oscillations, and the function containing time-periodic multipliers with frequencies $\nu_{1}, \nu_{2}$ :

$$
\begin{gathered}
H(\varphi, p, t)=H_{0}(\varphi, p)+F\left(\varphi, p, \tau_{1}, \tau_{2}\right), \quad H_{0}(\varphi, p)=\frac{p^{2}}{2 l}-g \cos \varphi \\
F\left(\varphi, p, \tau_{1}, \tau_{2}\right)=-\frac{a p \sin \varphi h_{1}\left(\tau_{1}\right)}{l}+\frac{a^{2} \sin ^{2} \varphi h_{1}^{2}\left(\tau_{1}\right)}{2 l}- \\
-\frac{b p \cos \varphi h_{2}\left(\tau_{2}\right)}{l}+\frac{b^{2} \cos ^{2} \varphi h_{2}^{2}\left(\tau_{2}\right)}{2 l}+\frac{a b \sin 2 \varphi h_{1}\left(\tau_{1}\right) h_{2}\left(\tau_{2}\right)}{2 l}, \quad \tau_{1}=\nu_{1} t, \tau_{2}=\nu_{2} t .
\end{gathered}
$$

We assume that $\nu_{1} \neq \nu_{2}$; otherwise we obtain a single-frequency system $\left(\tau_{1}=\tau_{2}=\tau\right)$ whose right-hand side can be averaged over the variable $\tau$ and for which we can use Remark 1 . The average value of the function $F\left(\varphi, p, \tau_{1}, \tau_{2}\right)$ over the variables $\tau_{1}$ and $\tau_{2}$ is

$$
\bar{F}(\varphi)=\frac{1}{s_{1} s_{2}} \int_{0}^{s_{1}} \int_{0}^{s_{2}} F\left(\varphi, p, \tau_{1}, \tau_{2}\right) d \tau_{1} d \tau_{2}=\frac{a^{2} \sin ^{2} \varphi \overline{h_{1}^{2}}}{2 l}+\frac{b^{2} \cos ^{2} \varphi \overline{h_{2}^{2}}}{2 l}
$$

where $s_{i}$ is the period of the function $h_{i}(\tau)$ and $\overline{h_{i}^{2}}=\frac{1}{s_{i}} \int_{0}^{s_{i}} h_{i}^{2}(\tau) d \tau, i=1,2$. Consequently, the Hamiltonian of the averaged system is

$$
\bar{H}(\varphi, p)=H_{0}(\varphi, p)+\bar{F}(\varphi)=\frac{p^{2}}{2 l}-g \cos \varphi+\frac{a^{2} \sin ^{2} \varphi \overline{h_{1}^{2}}}{2 l}+\frac{b^{2} \cos ^{2} \varphi \overline{h_{2}^{2}}}{2 l} .
$$

Let $\varphi\left(t, \nu_{1}, \nu_{2}\right), p\left(t, \nu_{1}, \nu_{2}\right)$ be the solution of the Hamilton equations with Hamiltonian (3.6) on the interval $0 \leqslant t \leqslant a(a>0)$, and let $\varphi^{*}(t), p^{*}(t)$ be the solution with the same initial conditions to the averaged Hamiltonian equations with Hamiltonian (3.7) on the interval $0 \leqslant t \leqslant a$. In accordance with Statement 3, we obtain an estimate of deviation of the solution to the averaged system from the solution to the initial system

$$
\left|\varphi\left(t, \nu_{1}, \nu_{2}\right)-\varphi^{*}(t)\right|+\left|p\left(t, \nu_{1}, \nu_{2}\right)-p^{*}(t)\right| \leqslant \frac{c_{1}}{\nu_{1}}+\frac{c_{2} \nu_{2}}{\nu_{1}}+\frac{c_{3}}{\nu_{2}} \quad \text { with } \quad 0 \leqslant t \leqslant a,
$$

where $c_{1}, c_{2}, c_{3}>0$ are some constants independent of $\nu_{1}, \nu_{2}$. Thus, the solutions of the initial system tend uniformly in $t$ to solutions of the averaged system if the oscillation frequencies $\nu_{1}, \nu_{2}$ tend to infinity and the ratio of frequencies $\frac{\nu_{2}}{\nu_{1}}$ tends to zero.

In some cases, one can obtain a more accurate estimate. Suppose, for example, that $h_{1}(\tau)=$ $=h_{2}(\tau)=\cos \tau$, i.e., the point of suspension of the pendulum undergoes oscillations according to the law

$$
x_{0}(t)=\frac{b}{\nu_{2}} \sin \nu_{2} t, \quad z_{0}(t)=\frac{a}{\nu_{1}} \sin \nu_{1} t, \quad \nu_{1} \neq \nu_{2} .
$$

Since

$$
h_{1}\left(\tau_{1}\right) h_{2}\left(\tau_{2}\right)=\cos \tau_{1} \cos \tau_{2}=\frac{1}{2}\left(\cos \left(\tau_{1}+\tau_{2}\right)+\cos \left(\tau_{1}-\tau_{2}\right)\right),
$$

the last term in the function $F\left(\varphi, p, \tau_{1}, \tau_{2}\right)$ can be represented as

$$
\frac{a b \sin 2 \varphi \cos \tau_{1} \cos \tau_{2}}{2 l}=\frac{a b \sin 2 \varphi \cos \tau_{3}}{4 l}+\frac{a b \sin 2 \varphi \cos \tau_{4}}{4 l},
$$


where $\tau_{3}=\left(\nu_{1}+\nu_{2}\right) t, \tau_{4}=\left(\nu_{1}-\nu_{2}\right) t$. Hence, in this case the Hamiltonian function is represented as a sum of the Hamiltonian function of the system unperturbed by oscillations, and four timeperiodic functions with frequencies $\nu_{1}, \nu_{2}, \nu_{1}+\nu_{2}, \nu_{1}-\nu_{2}$ :

$$
H(\varphi, p, t)=H_{0}(\varphi, p)+H_{1}\left(\varphi, p, \tau_{1}\right)+H_{2}\left(\varphi, p, \tau_{2}\right)+H_{3}\left(\varphi, \tau_{3}\right)+H_{4}\left(\varphi, \tau_{4}\right) .
$$

Therefore, according to Statement 2, we obtain an estimate of the deviation of the solution to the averaged system from the solution to the initial system

$$
\left|\varphi\left(t, \nu_{1}, \nu_{2}\right)-\varphi^{*}(t)\right|+\left|p\left(t, \nu_{1}, \nu_{2}\right)-p^{*}(t)\right| \leqslant \frac{c_{1}^{*}}{\nu_{1}}+\frac{c_{2}^{*}}{\nu_{2}}+\frac{c_{3}^{*}}{\nu_{1}+\nu_{2}}+\frac{c_{4}^{*}}{\left|\nu_{1}-\nu_{2}\right|} \quad \text { with } \quad 0 \leqslant t \leqslant a
$$

where $c_{1}^{*}, c_{2}^{*}, c_{3}^{*}, c_{4}^{*}>0$ are some constants independent of $\nu_{1}, \nu_{2}$. Thus, in this case the solutions of the initial system tend uniformly in $t$ to solutions of the averaged system if the oscillation frequencies $\nu_{1}, \nu_{2}$ and the absolute value of their difference tend to infinity. The last estimate improves the estimate (3.8). For example, for $\nu_{2}=2 \nu_{1}$ we have

$$
\left|\varphi\left(t, \nu_{1}, \nu_{2}\right)-\varphi^{*}(t)\right|+\left|p\left(t, \nu_{1}, \nu_{2}\right)-p^{*}(t)\right| \leqslant \frac{c}{\nu_{1}} \quad \text { with } \quad 0 \leqslant t \leqslant a,
$$

where $c$ is some constant independent of $\nu_{1}, \nu_{2}$.

Remark 3. According to (3.7), the potential energy $V^{*}(\varphi)$ of the averaged system is

$$
V^{*}(\varphi)=-g \cos \varphi+\frac{a^{2} \overline{h_{1}^{2}}-l^{2} b^{2} \overline{h_{2}^{2}}}{2 l} \sin ^{2} \varphi
$$

and coincides with the function (3.4). Therefore, the conclusions on the stability of the equilibrium points of the averaged system, as obtained in Example 1, hold in this example as well.

\section{Average values of functions on a torus for conditionally periodic motion}

Statement 5. Let $f\left(x_{1}, x_{2}\right)$ be a function that is continuously differentiable and periodic in the variables $x_{1}, x_{2}$ with period 1 . We assume it to be a function on a two-dimensional torus with angular coordinates $x_{1}, x_{2} \bmod 1$. Then for any conditionally periodic motion

$$
x_{1}=x_{1}^{0}+\omega_{1} \tau, \quad x_{2}=x_{2}^{0}+\omega_{2} \tau, \quad 0 \leqslant \tau<+\infty
$$

on this torus with frequencies $\omega_{1}, \omega_{2}$ the deviation of the time average from the space average for the function $f$ is estimated by the inequality

$$
\left|\lim _{T \rightarrow+\infty} \frac{1}{T} \int_{0}^{T} f\left(x_{1}^{0}+\omega_{1} \tau, x_{2}^{0}+\omega_{2} \tau\right) d \tau-\int_{0}^{1} \int_{0}^{1} f\left(x_{1}, x_{2}\right) d x_{1} d x_{2}\right| \leqslant c \frac{\omega_{2}}{\omega_{1}},
$$

where $c=L e^{L} / 2$ and $L$ is the Lipschitz constant of the function $f\left(x_{1}, x_{2}\right)$. 
Proof. Given $\nu_{1}>0, \nu_{2}>0, a>0$, consider the ordinary differential equation

$$
\frac{d u}{d t}=f\left(x_{1}^{0}+\nu_{1} t, x_{2}^{0}+\nu_{2} t\right), \quad u \in \mathbb{R}, \quad 0 \leqslant t \leqslant a .
$$

The averaged equation has the form

$$
\frac{d v}{d t}=g, \quad g=\int_{0}^{1} \int_{0}^{1} f\left(x_{1}, x_{2}\right) d x_{1} d x_{2}, \quad v \in \mathbb{R}, \quad 0 \leqslant t \leqslant a .
$$

Let $u\left(t, \nu_{1}, \nu_{2}\right)$ and $v(t)$ denote the solutions to Eqs. (4.2), (4.3) on the interval $0 \leqslant t \leqslant a$ with initial conditions $u\left(0, \nu_{1}, \nu_{2}\right)=0$ and $v(0)=0$ :

$$
u\left(t, \nu_{1}, \nu_{2}\right)=\int_{0}^{t} f\left(x_{1}^{0}+\nu_{1} s, x_{2}^{0}+\nu_{2} s\right) d s, \quad v(t)=g t .
$$

According to Statement 3, there are constants $c_{1}, c_{2}, c_{3}>0$ such that the estimate of the deviation of the solution $v(t)$ to the averaged equation from the solution $u\left(t, \nu_{1}, \nu_{2}\right)$ to the initial equation has the form

$$
\left|u\left(t, \nu_{1}, \nu_{2}\right)-v(t)\right| \leqslant \frac{c_{1}}{\nu_{1}}+\frac{c_{2} \nu_{2}}{\nu_{1}}+\frac{c_{3}}{\nu_{2}} \quad \text { with } \quad 0 \leqslant t \leqslant a .
$$

For $t=a$ we have

$$
\left|\int_{0}^{a} f\left(x_{1}^{0}+\nu_{1} s, x_{2}^{0}+\nu_{2} s\right) d s-g a\right| \leqslant \frac{c_{1}}{\nu_{1}}+\frac{c_{2} \nu_{2}}{\nu_{1}}+\frac{c_{3}}{\nu_{2}} .
$$

Taking any $T>0$ and rescaling time as $s=\frac{a \tau}{T}$ under the integral, we obtain

$$
\left|\frac{a}{T} \int_{0}^{T} f\left(x_{1}^{0}+\nu_{1} \frac{a \tau}{T}, x_{2}^{0}+\nu_{2} \frac{a \tau}{T}\right) d \tau-g a\right| \leqslant \frac{c_{1}}{\nu_{1}}+\frac{c_{2} \nu_{2}}{\nu_{1}}+\frac{c_{3}}{\nu_{2}} .
$$

Setting $\nu_{1}=\frac{T}{a} \omega_{1}, \nu_{2}=\frac{T}{a} \omega_{2}, a=1$, we obtain

$$
\left|\frac{1}{T} \int_{0}^{T} f\left(x_{1}^{0}+\omega_{1} \tau, x_{2}^{0}+\omega_{2} \tau\right) d \tau-g\right| \leqslant \frac{c_{1}}{T \omega_{1}}+\frac{c_{2} \omega_{2}}{\omega_{1}}+\frac{c_{3}}{T \omega_{2}} .
$$

Here $c_{2}=L e^{L} / 2$ according to (2.9). For $T \rightarrow+\infty$ we obtain inequality (4.1).

We note that, using inequality (2.16), one can obtain a similar statement for conditionally periodic motion on an $n$-dimensional torus. 
Statement 6. Let $f\left(x_{1}, \ldots, x_{n}\right)$ be a function that is continuously differentiable and periodic in the variables $x_{1}, \ldots, x_{n}$ with period 1 . We will assume it to be a function on an $n$-dimensional torus with angular coordinates $x_{1}, \ldots, x_{n} \bmod 1$. Then for any conditionally periodic motion

$$
x_{1}=x_{1}^{0}+\omega_{1} \tau, \ldots, x_{n}=x_{n}^{0}+\omega_{n} \tau, \quad 0 \leqslant \tau<+\infty
$$

on this torus with frequencies $\omega_{1}, \ldots, \omega_{n}$ the deviation of the time average from the space average for the function $f$ is estimated by the inequality

$$
\begin{gathered}
\left|\lim _{T \rightarrow+\infty} \frac{1}{T} \int_{0}^{T} f\left(x_{1}^{0}+\omega_{1} \tau, \ldots, x_{2}^{0}+\omega_{2} \tau\right) d \tau-\int_{0}^{1} \ldots \int_{0}^{1} f\left(x_{1}, \ldots, x_{n}\right) d x_{1} \ldots d x_{n}\right| \leqslant \\
\leqslant c\left(\frac{\omega_{2}}{\omega_{1}}+\ldots+\frac{\omega_{n}}{\omega_{n-1}}\right),
\end{gathered}
$$

where $c=L e^{L} / 2$ and $L$ is the Lipschitz constant of the function $f$.

\section{References}

[1] Kugushev, E.I., Levin, M. A., and Popova, T.V., Holonomic Systems on a Rapidly Oscillating Platform, J. Appl. Math. Mech., 2017, vol.81, no. 5, pp. 360-367; see also: Prikl. Mat. Mekh., 2009, vol. 81, no. 5, pp. 523-533.

[2] Bogolubov, N. N. and Mitropolskiy, Yu. A., Asymptotic Methods in the Theory of Nonlinear Oscillations, Moscow: Nauka, 1974 (Russian).

[3] Arnol'd, V.I., Kozlov, V.V., and Neřshtadt, A. I., Mathematical Aspects of Classical and Celestial Mechanics, 3rd ed., Encyclopaedia Math. Sci., vol. 3, Berlin: Springer, 2006.

[4] Grebenikov, E. A., Mitropolskiy, Yu. A., and Ryabov, Yu. A., Introduction to Resonance Analytical Dynamics, Moscow: Yanus-K, 1999 (Russian).

[5] Zhuravlev, V.F. and Klimov, D. M., Applied Methods in Vibration Theory, Moscow: Nauka, 1988 (Russian).

[6] Hapaev, M.M., Averaging in Stability Theory: A Study of Resonance Multi-Frequency Systems, Math. Appl., vol. 79, Dordrecht: Springer, 1993.

[7] Bakhtin, V.I., Averaging in Multifrequency Systems, Funct. Anal. Appl., 1986, vol. 20, no.2, pp. 83-88; see also: Funktsional. Anal. i Prilozhen., 1986, vol.20, no. 2, pp.1-7.

[8] Krasil'nikov, P.S., Applied Methods of Investigation of Nonlinear Oscillations, Izhevsk: R\&C Dynamics, Institute of Computer Science, 2015 (Russian).

[9] Arnol'd, V.I., Geometrical Methods in the Theory of Ordinary Differential Equations, 2nd ed., Grundlehren Math. Wiss., vol. 250, New York: Springer, 1988.

[10] Stephenson, A., On a New Type of Dynamical Stability, Mem. Proc. Manch. Lit. Phil. Soc., 1908, vol. 52, no. 8, pp. 1-10.

[11] Kapitza, P. L., Pendulum with a Vibrating Suspension, Usp. Fiz. Nauk, 1951, vol.44, pp.7-15 (Russian). See also: Collected Papers of P. L. Kapitza: Vol. 2, D. ter Haar (Ed.), Oxford: Pergamon, 1965, pp. 726-732,732a,732b,733-737.

[12] Kapitza, P. L., Dynamical Stability of a Pendulum When Its Point of Suspension Vibrates, Zh. Èksp. Teor. Fiz., 1951, vol. 21, no. 5, pp. 588-597 (Russian). See also: Collected Papers of P. L. Kapitza: Vol. 2, D. ter Haar (Ed.), Oxford: Pergamon, 1965, pp. 714-725.

[13] Strizhak, T. G., Methods of Investigation in Dynamical Systems of Pendulum-Type, Alma-Ata: Nauka, 1981 (Russian).

[14] Blekhman, I. I., Vibrational Mechanics, Moscow: Nauka, 1994 (Russian). 
[15] Kholostova, O. V., Problems of Dynamics of Solids with Vibrating Suspension, Izhevsk: R\&C Dynamics, Institute of Computer Science, 2016 (Russian).

[16] Markeev, A. P., On the Theory of Motion of a Rigid Body with a Vibrating Suspension, Dokl. Phys., 2009, vol.54, no.8, pp. 392-396; see also: Dokl. Akad. Nauk, 2009, vol.427, no.6, pp. 771-775.

[17] Markeev, A.P., On the Motion of a Heavy Dynamically Symmetric Rigid Body with Vibrating Suspension Point, Mech. Solids, 2012, vol.47, no.4, pp.373-379; see also: Izv. Akad. Nauk. Mekh. Tverd. Tela, 2012, no. 4, pp. 3-10.

[18] Petrov, A. G., Vibratory Energy of a Conservative Mechanical System, Dokl. Phys., 2010, vol.55, no. 4, pp. 203-206; see also: Dokl. Akad. Nauk, 2010, vol.431, no. 6, pp. 762-765.

[19] Zhuravlev, V.F., Petrov, A. G., and Shunderyuk, M. M., Selected Problems of Hamiltonian Mechanics, Moscow: Lenand, 2015 (Russian).

[20] Markeev, A.P., On the Accuracy Problem for Pendulum Clock on a Vibrating Base, Mech. Solids, 2018, vol.53, no.5, pp.573-583; see also: Izv. Akad. Nauk. Mekh. Tverd. Tela, 2018, no.5, pp. 112-123.

[21] Neishtadt, A. I. and Sheng, K., Bifurcations of Phase Portraits of Pendulum with Vibrating Suspension Point, Commun. Nonlinear Sci. Numer. Simul., 2017, vol.47, pp. 71-80. 